\title{
Shortcomings of SARS-CoV-2 genomic metadata
}

Landen Gozashti ${ }^{12^{*}}$

1'Department of Organismic and Evolutionary Biology and Museum of Comparative Zoology, Harvard University, Cambridge, MA, 02138, USA

${ }^{2}$ Department of Biomolecular Engineering and Genomics Institute, University of California Santa Cruz. Santa Cruz, CA 95064, USA

*Igozashti@g.harvard.edu

\begin{abstract}
:
Metadata is integral to data-driven association studies relevant to epidemiology, viral population dynamics and public health response. However, SARS-CoV-2 metadata quality remains inadequate. Here I exemplify this through a brief analysis of two metadata categories in the GISAID SARS-CoV-2 genomic database: "originating lab" and "submitting lab". My analysis reveals a startling prevalence of spelling errors and inconsistent naming conventions, which together occur in an estimated $\sim 9.8 \%$ and $\sim 11.6 \%$ of "originating labs" and "submitting labs" respectively. In addition, I find numerous ambiguous lab names, such as "Biology Dpt" and "Hospital" which provide very little information with regard to the actual source of a sample and could easily associate with multiple sources worldwide. Importantly, all of these issues can impair the ability and accuracy of association studies by deceptively causing a group of samples to identify with multiple sources when they truly all identify with one source, or vice versa. GISAID's "originating lab" and "submitting lab" categories are specifically relevant to identifying problematic sites in SARS-CoV-2 genomic data through lab association. Thus, I advocate that both data submitters and maintainers strive for a higher metadata quality standard now and in the future.
\end{abstract}

\section{Main}

Metadata, or "data about data," ${ }^{1}$ is an essential component of science: informing both data-driven analyses and decisions with regards to public health ${ }^{2,3}$. In spite of this, metadata standards remain somewhat neglected ${ }^{4-6}$. As some groups have previously mentioned, the COVID-19 pandemic has shed light on this, and metadata inadequacies have hindered association studies relevant to both epidemiology and viral population dynamics ${ }^{7-9}$. Databases such as GISAID ${ }^{10}$ and Nextstrain ${ }^{11}$ have empowered copious 
SARS-CoV-2 studies by maintaining SARS-CoV-2 genomic sequences and corresponding metadata. Here, I specifically highlight inconsistencies in "originating lab" and "submitting lab" descriptions within the GISAID database, which maintained 223,024 SARS-CoV-2 genomic sequences as of November 27th 2020, to exemplify where improvements in metadata quality are needed and to raise awareness to data submitters and maintainers alike.

I used a manual string comparison approach to estimate the prevalence of spelling errors and naming inconsistencies in "originating lab" and "submitting lab" metadata categories for all GISAID SARS-CoV-2 sequences as of November 27th 2020. My analysis reveals that an alarmingly large proportion of lab names are misspelled or exhibit inconsistent naming conventions among samples at least once: $\sim 9.8 \%$ and $\sim 11.6 \%$ for "originating labs" and "submitting labs" respectively. Furthermore, I observe many instances in which lab names are misspelled or named inconsistently multiple times across samples, and cases of highly ambiguous lab names such as "Hospital" or "Biology Dpt" that could be associated with multiple sources (Figure 1A,B). One of the primary consequences of spelling errors and inconsistent naming conventions in these particular categories (and more generally) is the appearance that a group of samples identifies with multiple sources, when they all truly identify with one particular source (Figure 1C). One consequence of ambiguity is the opposite effect, in which several samples all identify with the same source when in truth those samples all stem from different sources. Both of these deceptions can impair association studies. Notably, "originating lab" and "submitting lab" metadata categories are pertinent to the ability to accurately identify problematic sites in SARS-CoV-2 genomes and the sources and causes of erroneous variants in SARS-CoV-2 genomic data ${ }^{8,12}$. Thus, I urge producers of SARS-CoV-2 genomic data to proceed with caution when submitting their metadata, and advocate that maintainers of genomic databases be aware of possible errors in incoming metadata (such as those I show) and attentively promote metadata standardization. On the other hand, the development of new reliable methods for SARS-CoV-2 metadata correction could serve as an alternative solution and could likely be applied across multiple disciplines ${ }^{1,13,14}$.

The SARS-CoV-2 pandemic has prompted an unprecedented response from the scientific and public health community, and the development and maintenance of databases such as GISAID have permitted epidemiological and comparative studies of unparalleled power. However, a brief analysis reveals that the quality of metadata accompanying such datasets remains unreliable. The importance of quality metadata with regard to our ability as a species to combat this pandemic and future pandemics is now more important than ever, and we must strive for a higher standard. 


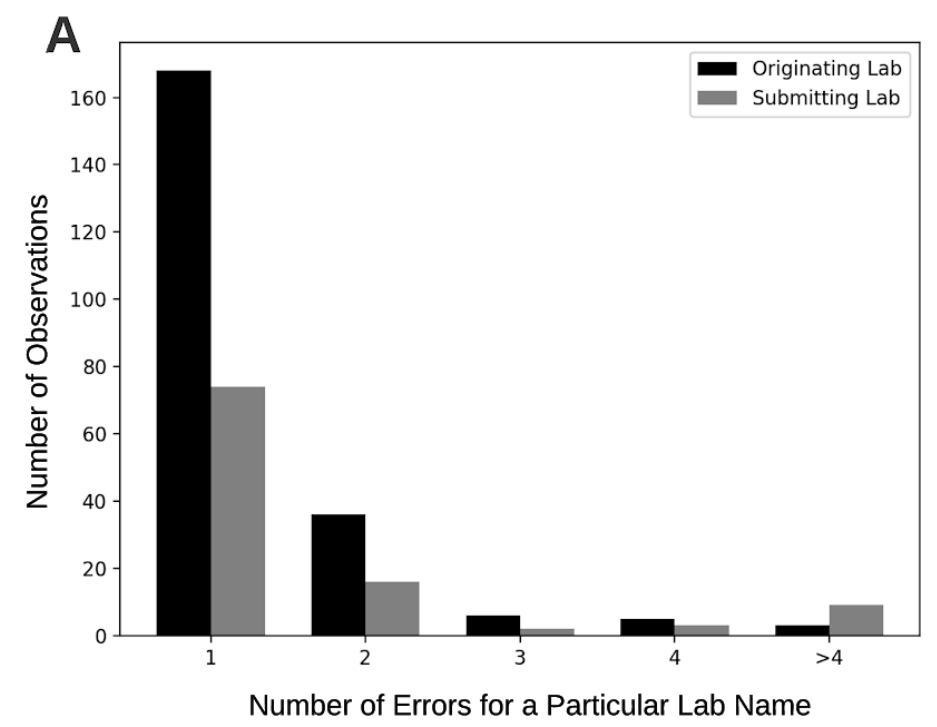

B
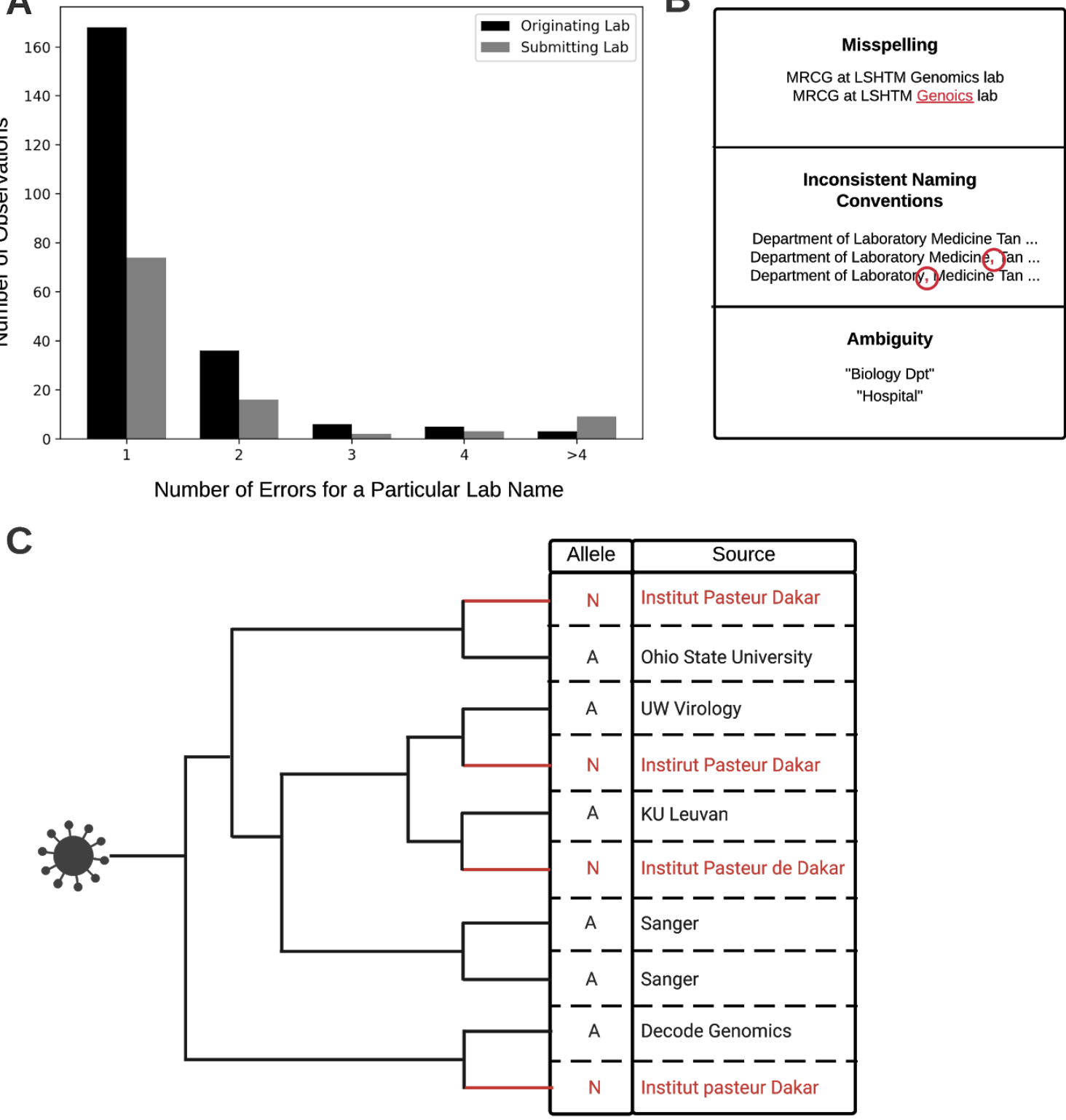

Figure 1: (A) A bar plot displaying the number of times I observe 1, 2, 3, 4 and $>4$ errors for a given lab name in the "Originating Lab" and "Submitting Lab" metadata categories in the GISAID database as of 27 November 2020, accompanied by (B) some observed examples of misspellings, inconsistent naming conventions, and highly ambiguous entries. (C) A phylogenetic tree displaying an example of a case in which errors in "originating lab" metadata might impede association studies with regard to SARS-CoV-2 genomic data. In this case, ambiguous "N" alleles occur multiple times across a phylogeny at a given site and all stem from the same source. However, metadata errors (shown in red) cause this ambiguous "N" allele to appear as if it is associated with 4 different sources (rather than 1). Such a site could impair phylogenetic inference and 
should be flagged in the SARS-CoV-2 masking recommendations but could be overlooked as a result of these errors ${ }^{12}$.

\section{Acknowledgements}

The author thanks the GISAID database and all labs who contributed SARS-CoV-2 sequence data. I also thank Scott W. Roy, Bryan Thornlow and Russell Corbett-Detig for feedback.

\section{References}

1. Goble, C., Corcho, O., Alper, P. \& De Roure, D. e-Science and the Semantic Web: A Symbiotic Relationship. in Discovery Science 1-12 (Springer Berlin Heidelberg, 2006).

2. Matters, M. D., Lekiachvili, A., Savel, T. \& Zheng, Z.-J. Developing metadata to organize public health datasets. AMIA Annu. Symp. Proc. 1047 (2005).

3. Field, D. et al. The minimum information about a genome sequence (MIGS) specification. Nat. Biotechnol. 26, 541-547 (2008).

4. Fabreau, G. E., Minty, E. P., Southern, D. A., Quan, H. \& Ghali, W. A. A Meta-Data Manifesto: The Need for Global Health Meta-Data. Int J Popul Data Sci 3, 436 (2018).

5. Field, D. et al. The Genomic Standards Consortium. PLoS Biol. 9, e1001088 (2011).

6. Wilkinson, M. D. et al. The FAIR Guiding Principles for scientific data management and stewardship. Sci Data 3, 160018 (2016).

7. Kaiser, K. A. et al. Metadata: The accelerant we need. Inf. Serv. Use 40, 181-191 (2020).

8. Turakhia, Y. et al. Stability of SARS-CoV-2 phylogenies. PLoS Genet. 16, 
e1009175 (2020).

9. Schriml, L. M. et al. COVID-19 pandemic reveals the peril of ignoring metadata standards. Sci Data 7, 188 (2020).

10. Shu, Y. \& McCauley, J. GISAID: Global initiative on sharing all influenza data - from vision to reality. Euro Surveill. 22, (2017).

11. Hadfield, J. et al. Nextstrain: real-time tracking of pathogen evolution. Bioinformatics 34, 4121-4123 (2018).

12. De Maio, N. et al. Issues with SARS-CoV-2 sequencing data. (2020).

13. Michener, W. K. Ecological data sharing. Ecol. Inform. 29, 33-44 (2015).

14. Assaf, A., Senart, A. \& Troncy, R. Roomba: Automatic Validation, Correction and Generation of Dataset Metadata. in Proceedings of the 24th International Conference on World Wide Web 159-162 (Association for Computing Machinery, 2015). 